\title{
Article \\ Insertion of the Liquid Crystal 5CB into Monovacancy Graphene
}

\author{
Paul A. Brown ${ }^{1}$, Jakub Kołacz ${ }^{2}$, Sean A. Fischer ${ }^{1}$, Christopher M. Spillmann ${ }^{2} \odot$ and Daniel Gunlycke ${ }^{1, *}$ \\ 1 Chemistry Division, United States Naval Research Laboratory, Washington, DC 20375, USA; \\ paul.brown@nrl.navy.mil (P.A.B.); safischer012@gmail.com (S.A.F.) \\ 2 Center for Bio/Molecular Science and Engineering, United States Naval Research Laboratory, \\ Washington, DC 20375, USA; jakub.kolacz@nrl.navy.mil (J.K.); christopher.spillmann@nrl.navy.mil (C.M.S.) \\ * Correspondence: daniel.gunlycke@nrl.navy.mil
}

Citation: Brown, P.A.; Kołacz, J.;

Fischer, S.A.; Spillmann, C.M.;

Gunlycke, D. Insertion of the Liquid

Crystal 5CB into Monovacancy

Graphene. Molecules 2022, 27, 1664.

https://doi.org/10.3390/

molecules27051664

Academic Editors: Pradip

K. Bhowmik and

Alfonso Martinez-Felipe

Received: 13 January 2022

Accepted: 26 February 2022

Published: 3 March 2022

Publisher's Note: MDPI stays neutral with regard to jurisdictional claims in published maps and institutional affiliations.

Copyright: (๑) 2022 by the authors Licensee MDPI, Basel, Switzerland. This article is an open access article distributed under the terms and conditions of the Creative Commons Attribution (CC BY) license (https:// creativecommons.org/licenses/by/ $4.0 /)$.

\begin{abstract}
Interfacial interactions between liquid crystal (LC) and two-dimensional (2D) materials provide a platform to facilitate novel optical and electronic material properties. These interactions are uniquely sensitive to the local energy landscape of the atomically thick 2D surface, which can be strongly influenced by defects that are introduced, either by design or as a byproduct of fabrication processes. Herein, we present density functional theory (DFT) calculations of the LC mesogen 4cyan-4'-pentylbiphenyl (5CB) on graphene in the presence of a monovacancy (MV-G). We find that the monovacancy strengthens the binding of $5 \mathrm{CB}$ in the planar alignment and that the structure is lower in energy than the corresponding homeotropic structure. However, if the molecule is able to approach the monovacancy homeotropically, 5CB undergoes a chemical reaction, releasing $4.5 \mathrm{eV}$ in the process. This reaction follows a step-by-step process gradually adding bonds, inserting the $5 \mathrm{CB}$ cyano group into MV-G. We conclude that this irreversible insertion reaction is likely spontaneous, potentially providing a new avenue for controlling both LC behavior and graphene properties.
\end{abstract}

Keywords: liquid crystal; graphene; defect; monovacancy

\section{Introduction}

Liquid crystal (LC) on atomically thin 2D interfaces results in unique behaviors such as surface-induced phase transitions [1], tilt angle control [2], selective alignment [3], and ion trapping [4]. While research on integrated LC-2D material systems is still in its infancy, their properties have been exploited to image and characterize 2D materials using LC to optically transduce surface defects [5], domains [6,7], degradation [8] and the number of 2D layers [2]. Conductive 2D materials such as graphene have also been demonstrated as a single-component electrode and alignment layer $[9,10]$. The large effect of atomic-level interactions demonstrates the importance of $2 \mathrm{D}$ surfaces on further miniaturization of LC electrooptic devices, leading to smaller form factors, faster response times [11] and new behaviors.

Defect engineering of 2D materials is increasingly becoming feasible on the atomic scale [12-14]. Atomic-scale control is a critical challenge for nanoscale development of new materials for nascent technologies. Research on 2D materials have expanded rapidly [15-18] and influenced electronics [19,20], catalysis [21-23], energy storage [24], batteries [25], topotronics [26,27], sensing [28] and photonics [29-32]. Moreover, 2D materials, including graphene, provide a large surface area for anchoring of atoms or molecules introduced into the system. Furthermore, advances in nanoscale patterning and defect engineering raise the prospect of incorporating and controlling liquid crystals on such surfaces [33,34]. 2D materials could thus facilitate exceptional binding of mesogen compounds, especially compounds whose chemical structure resembles the underlying 2D material itself as such compounds could, e.g., bind through $\pi-\pi$ stacking [35-38]. In addition, we expect that defects will play a central role in future applications, as they can provide both control and anchoring sites for absorbates. 
Defects often arise naturally in 2D materials during fabrication, including monovacancies, divacancies, Stone-Thrower-Wales defects, line defects, grain boundaries and impurities [39-49]. Herein, we focus on monovacancy graphene (MV-G), as graphene is a well-studied and easily fabricated 2D material and the monovacancy is a common and simple intrinsic point defect, acting as a reactive center for binding atomic or molecular matter [21-23,48,50-52]. Despite this, the attention in the field has so far focused on the properties of LC on pristine nanosheets [36-38], with little consideration of the potential reactivity of defects.

In this article, we present DFT calculations exploring the possibility that 5CB chemically reacts with a monovacancy in MV-G and under what circumstances such reactions take place. To probe this reactivity, we consider MV-G with three 5CB anchoring alignments: planar, homeotropic and pre-tilt. Our analyses include nudge elastic band (NEB) calculations to determine the reaction pathway and projected density of states (PDOS) calculations to identify key atoms involved in the insertion process. Lastly, we discuss the source of the reactivity in homeotropically aligned 5CB.

\section{Results and Discussion}

\section{1. $5 \mathrm{CB} / \mathrm{MV}-\mathrm{G}$ Interaction}

We begin by considering the equilibrium structure of $5 \mathrm{CB}$ adsorbed onto $\mathrm{MV}-\mathrm{G}$ in the planar alignment. In this case, the equilibrium distance is $3.34 \AA$ with a binding energy of $-1.60 \mathrm{eV}$ (Figure 1a). The binding energy of $5 \mathrm{CB}$ onto MV-G is $0.29 \mathrm{eV}$ lower than that for the corresponding $5 \mathrm{CB}$ binding energy $-1.31 \mathrm{eV}$ onto graphene without the monovacancy [38]. The adsorption energy can be viewed as a molecular analog to surface anchoring energy for the LC, suggesting a stronger surface anchoring energy in the presence of the monovacancy.
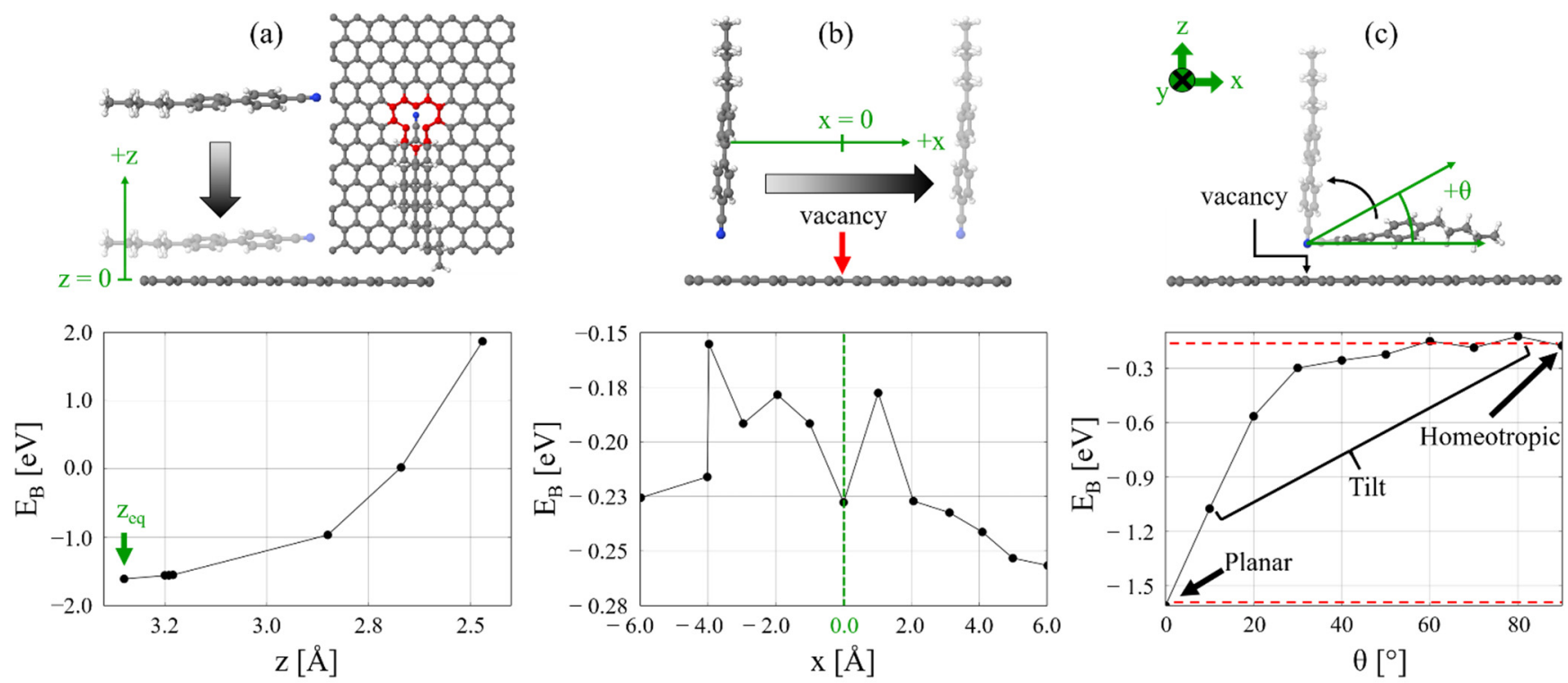

Figure 1. Binding energy scans. (a) Planar scan starting at the equilibrium distance $z_{\text {eq }}$. (b) Homeotropic scan relative to the monovacancy position. (c) Pre-tilt scan with dashed red lines marking the planar and monotonicity of the binding energy.

To probe the possibility of chemical binding, we explored 5CB in the planar orientation being compressed toward the monovacancy. The cyano substituent is oriented with the cyano-carbon above two of the carbon sites immediately adjacent to the Jahn-Tellerdistorted monovacancy and the cyano-nitrogen above the third adjacent monovacancy carbon atom. The reason for this orientation is that the cyano group, which is a highly polarized substituent and responsible for the dipolar character of 5CB, exposes the partially positive cyano-carbon to the dangling bonds of the monovacancy carbon atoms, 
maximizing the likelihood to induce a reactive event. This commonly occurs in reductive elimination reactions of cyano functional groups [53,54]. As is shown in Figure 1a, however, the computed binding energy increases monotonically as 5CB approaches, suggesting that there is no chemical bonding between the $5 \mathrm{CB}$ and the monovacancy in the planar orientation. Not shown in this figure is that the compression of 5CB onto MV-G also induces stress, causing a single monovacancy carbon atom to protrude out-of-plane, away from the $5 \mathrm{CB}$. The two Jahn-Teller carbons flex toward 5CB, further indicating that the possibility of planar attachment to the monovacancy is unlikely.

Next, we consider the homeotropic alignment to probe for potential reactivity (Figure 1b). Overall, a similar pattern of the binding energetics emerges for homeotropic alignment of $5 \mathrm{CB}$ compared to the planar case though with some noticeable differences. The lowest binding energy is $-0.26 \mathrm{eV}$ when the lateral in-plane distance between the 5CB cyano substituent and the monovacancy is $6.0 \AA$. We also observe a relatively low adsorption energy between $-4.0 \AA$ and $2.0 \AA$. Above the monovacancy, the binding energy is $-0.23 \mathrm{eV}$. Though the planar binding energy is lower than the corresponding the homeotropic binding energy, steric effects make chemical binding unlikely. Instead, we expect that the homeotropic alignment is more conducive to chemical binding.

We also performed an angular scan between the planar and homeotropic orientations. As Figure 1c shows, the binding energy increases linearly between $0-20^{\circ}$ and then saturates at approximately $-0.20 \mathrm{eV}$ approaching the homeotropic state. This analysis indicates that for partial coverage of $5 \mathrm{CB}$ on the graphene surface, approximately $1.4 \mathrm{eV}$ is required for the molecule to reach homeotropic alignment above the monovacancy. However, because the binding energy saturates near $30^{\circ}$, homeotropic alignment could also easily be reached for a 5CB molecule approaching MV-G within a large range of angles around normal incidence.

To understand the potential reactivity between $5 \mathrm{CB}$ and the carbon vacancy site, $5 \mathrm{CB}$ is placed in the homeotropic state directly above MV-G and allowed to relax into the monovacancy site (Figure 2a). The initial electron density (Figure 2b) is independently localized on the Jahn-Teller distorted monovacancy and on the cyano head of the 5CB molecule. As the molecule approaches the surface, the cyano head inserts itself into the defect, stabilizing the structure and causing the biphenyl core to twist (Figure 2c). The final structure exhibits one hexagonal and two non-hexagonal rings (Figure 2d) $[55,56]$ that tilt out of the plane at the cyano-nitrogen $(N)$, cyano-carbon $\left(C_{N}\right)$ and first carbon $\left(C_{1}\right)$.
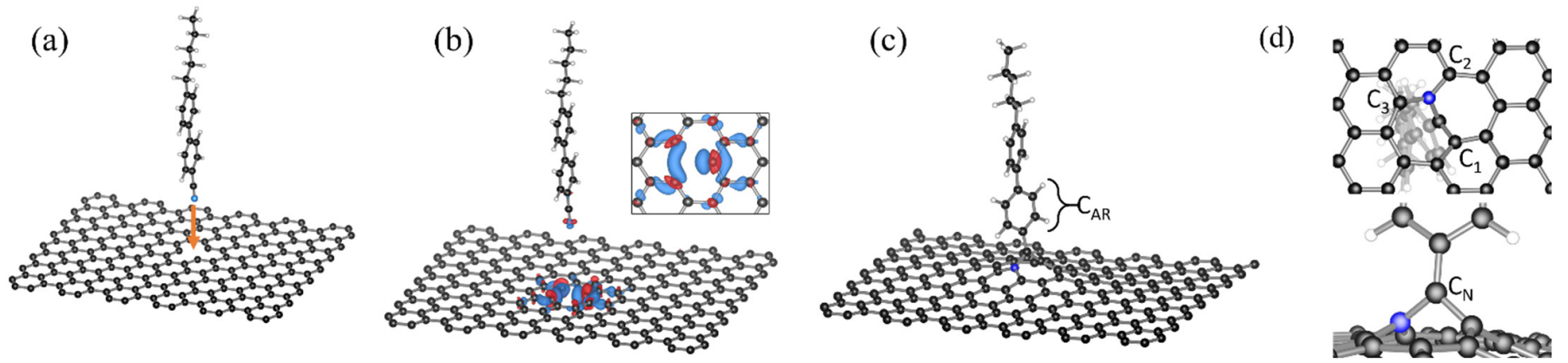

Figure 2. (a) 5CB homeotropically aligned above the monovacancy in MV-G. (b) Spin-polarized density of $5 \mathrm{CB} / \mathrm{MV}-\mathrm{G}$ with the inset showing a planar view of the corresponding density for the Jahn-Teller-distorted MV-G. (c,d) Images of the inserted 5CB molecule. The cyano-nitrogen (N) has filled the monovacancy and reestablished the hexagonal structure of graphene (upper panel in (d)), with the exception that the cyano-carbon $\left(\mathrm{C}_{\mathrm{N}}\right)$ has formed a bridge between $\mathrm{N}$ and the carbon site $\left(C_{1}\right)$. The bridge chemically binds the aromatic carbons $\left(C_{A R}\right)$ and the rest of the $5 C B$ molecule to the surface.

An analysis of the spin-polarized band structures (Figure 3a) shows that the presence of 5CB in the planar state near a monovacancy has only a small effect on graphene's electronic properties. The slight p-doping of the graphene, as is shown by the shift in the 
Dirac point, occurs due to Jahn-Teller distortions in MV-G and is observed even without the presence of 5CB $[57,58]$. The small HOMO-LUMO energy gap is also consistent with values for MV-G [59]. The DOS exhibits symmetry in the spin states of the 5CB molecule (N, $\mathrm{C}_{\mathrm{N}}$ and $\mathrm{C}_{\mathrm{AR}}$ peaks), which means the molecule remains in a non-magnetic state with a DOS similar to that of $5 \mathrm{CB}$ on pristine graphene [38], while the MV-G itself is in a magnetic state.
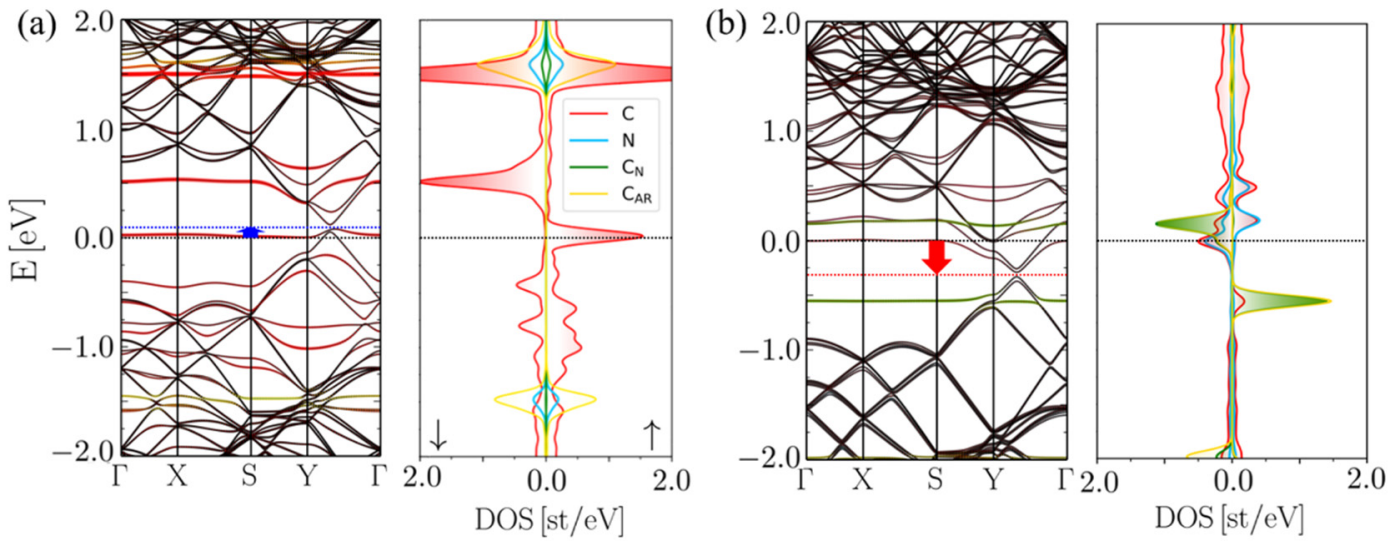

Figure 3. Spin-polarized band structures and density of states plots of (a) planar and (b) homeotropically inserted 5CB/MV-G. The blue and red arrows in the band structures indicate $\mathrm{p}$ - and $\mathrm{n}$-doping of the graphene, respectively. Spin up (positive) and spin down (negative) are highlighted in the right panels.

In the case where homeotropic 5CB comes into contact with the monovacancy, the insertion reaction results in a mixing of the orbitals that induces a magnetic state in the $5 C B$ constituent (Figure 3b). This is evidenced by the different DOS peaks localized at the $5 \mathrm{CB}$ sites for spin up and down. The magnetic moment is around $1.5 \mu_{\mathrm{B}}$. The $-260 \mathrm{meV}$ shift in the Dirac point results in n-doped graphene. Furthermore, the band structure of inserted 5CB/MV-G shows strong orbital mixing in bands near the Fermi energy from the cyano-carbon, cyano-nitrogen, aromatic carbons and monovacancy carbon atoms.

\subsection{Insertion Reaction}

An NEB calculation provides insight into the reaction path for $5 \mathrm{CB}$ insertion into MV-G. The energy analysis is shown in Figure 4a (The corresponding structures and PDOS can be found in the Supplementary Materials in Figures S1 and S2, respectively). The first four data points (reaction coordinates 1 through 4) show the total energy during the initial approach of $5 \mathrm{CB}$ to the monovacancy, starting at a distance of $5.25 \AA$ and ending at $1.61 \AA$ above the nanosheet. Note that all our calculations in this region produce non-magnetic solutions. Any potential magnetic solution, however, is expected to be close in energy, as past DFT calculations on graphene with a monovacancy without 5 CB suggests that the largest energy difference between a magnetic and the non-magnetic solution is of the order $3 \times 10^{-2} \mathrm{eV}$ [60]. As 5CB reaches its physisorbed state at $1.61 \AA$ above the monovacancy the overall energy lowers by nearly $-7 \times 10^{-2} \mathrm{eV}$ (Figure 4 inset). The lowered energy suggests that initial $5 \mathrm{CB}$ insertion into the monovacancy is stabilized. 


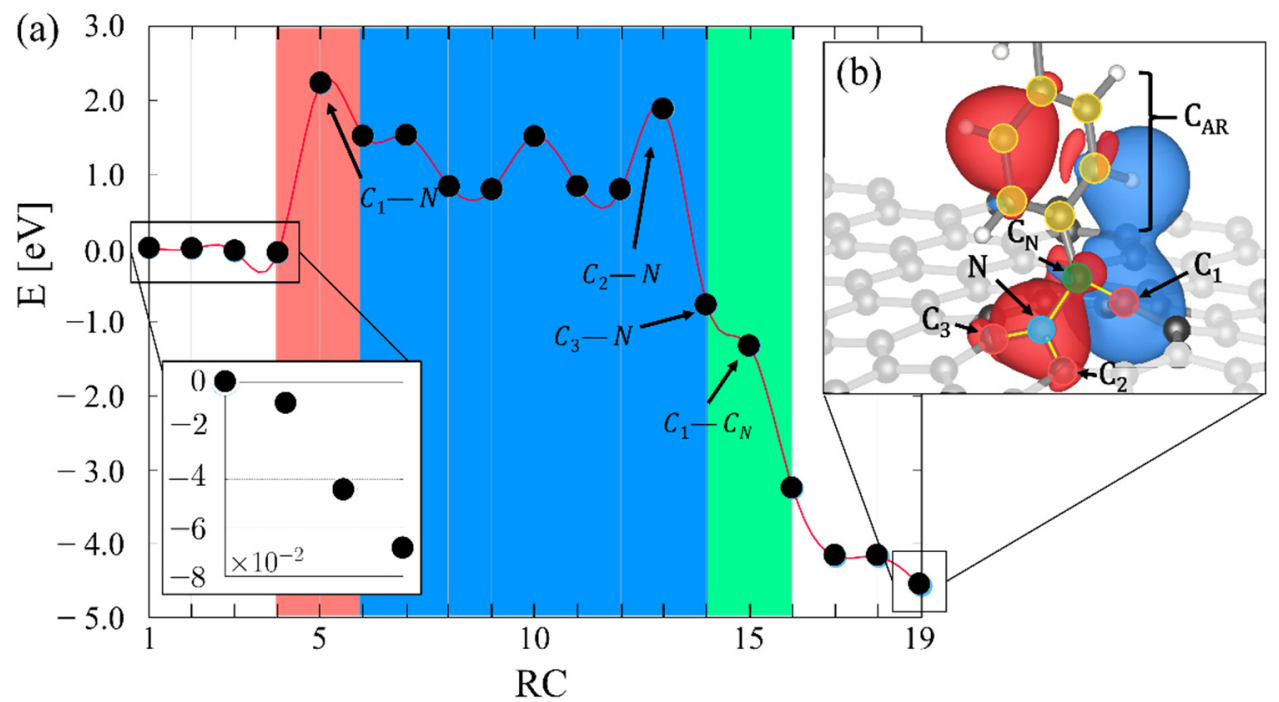

Figure 4. (a) Reaction path for the reaction coordinate (RC) of 5CB homeotropic insertion. The colored background marks the important chemical events along the reaction path beginning with insertion (red), tetrahedral formation (blue) and cyano-carbon reorganization (green). Inset shows the initial approach. (b) Spin-polarized charge density difference $\left(\Delta \rho\left[\mathrm{bohr}^{-1}\right]\right)$ of the final state.

The initial barrier along the reaction path is approximately $2.2 \mathrm{eV}$ (Figure $4 \mathrm{a}$, red shading), which is consistent with reported energy barriers for insertion of mon- and diatomic species into monovacancy graphene [61,62]. This region marks the initial insertion of the cyano head into the monovacancy. The energy of the system remains high as the $\mathrm{N}$ site bonds in turn to $C_{1}, C_{2}$ and $C_{3}$, as shown by the blue region in Figure 4a. Once the $\mathrm{N}$ bonding is complete, the four sites $\mathrm{N}, \mathrm{C}_{1}, \mathrm{C}_{2}$ and $\mathrm{C}_{3}$ form an sp ${ }^{3}$-hybridized tetrahedral structure that lowers the energy significantly. The green region of Figure 4a highlights the steps where $C_{1}$ bonds first to $C_{N}$ and then detaches from the nitrogen, creating a bridge between $C_{1}$ and $N$. This stage allows all relevant sites, including $N, C_{N}$ and $C_{1}$ to become $\mathrm{sp}^{2}$-hybridized, which results in a further energy reduction. The last step of the reaction twists the biphenyl of the $5 \mathrm{CB}$, reducing the delocalization of charge in the core of the molecule [37]. The final structure is $4.5 \mathrm{eV}$ lower in energy than the initial structure and the residual spin-polarized charge density difference in Figure $4 \mathrm{~b}$ includes sites across the phenyl ring (aromatic carbons in yellow) extending to the monovacancy in MV-G.

To highlight the salient steps in the reaction path, Figure 5 shows the PDOS of the monovacancy carbons, cyano-substituents and aromatic carbons at certain steps in the reaction. First, charge mixing appears between the cyano substituent and the monovacancy (Figure 5a), initially forming an $\mathrm{N}-\mathrm{C}_{1}$ bond. At this stage, the charge density is localized around the carbon atoms in the monovacancy. After the first bond is formed, there is a peak in the DOS at the fermi energy stemming from the aromatic carbons in the 5CB molecule (yellow peak in Figure 5b). The appearance of this peak suggests that delocalized charge in the phenyl ring plays a role in the charge reorganization that leads to $\mathrm{N}-\mathrm{C}_{3}$ bond formation. Finally, when the last tetrahedral nitrogen bond is formed at $\mathrm{N}-\mathrm{C}_{3}$, the dominant DOS contribution near the Fermi energy is a result of $C_{N}$ (Figure $5 c$ ). The tetrahedral structure is evident. At this point, there is only a single bond holding the cyano-group together. Note the increased $\mathrm{N}-\mathrm{C}_{\mathrm{N}}$ bond length in Figure $5 \mathrm{c}$ compared to that in Figure $5 \mathrm{~b}$. This sets the stage for the final bond reorganization that connects the under-coordinated $\mathrm{C}_{\mathrm{N}}$ with the monovacancy at $C_{1}$, leading to the final structure shown in Figure $4 \mathrm{~b}$. 

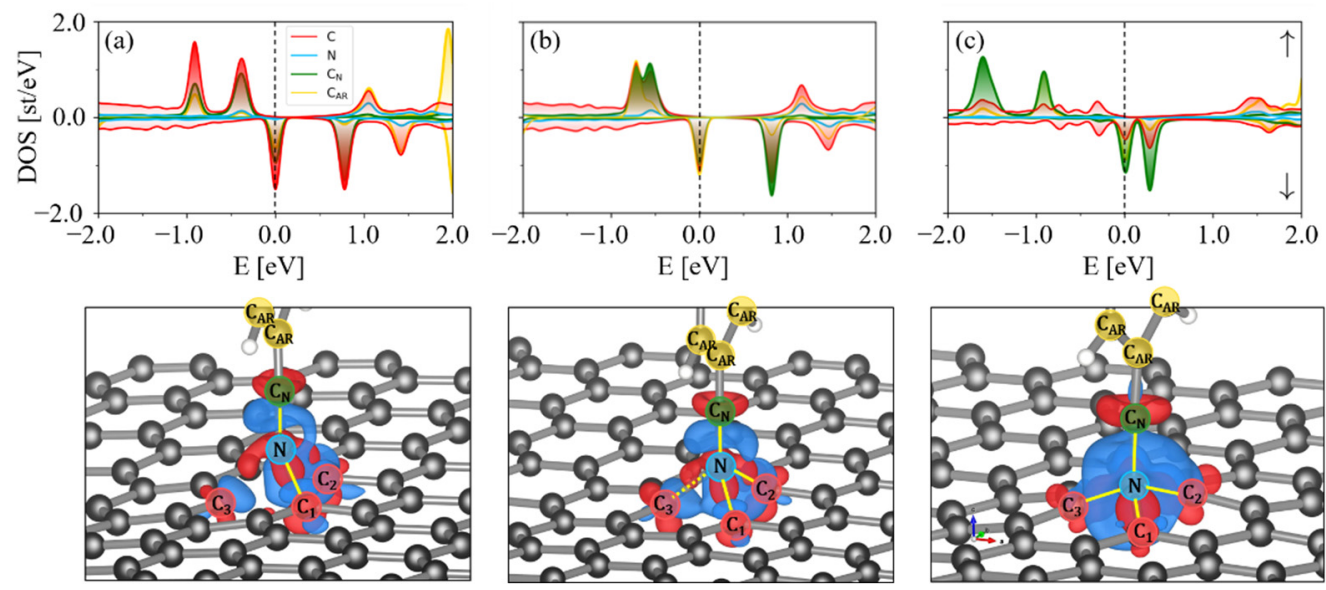

Figure 5. PDOS and structures during tetrahedral nitrogen formation from Figure 3. (a) $\mathrm{C}_{1}-\mathrm{N}$ bond formed at $\mathrm{RC}=5$. (b) $\mathrm{C}_{2}-\mathrm{N}$ bond formed, with a partial $\mathrm{C}_{3}-\mathrm{N}$ bond beginning to form at $\mathrm{RC}=13$. (c) $\mathrm{C}_{3}-\mathrm{N}$ bond fully formed at $\mathrm{RC}=14$. The color scheme is as follows: (red) vacancy carbon atoms, (blue) cyano-nitrogen $(\mathrm{N})$, (green) cyano-carbon $\left(\mathrm{C}_{\mathrm{N}}\right)$ and (yellow) aromatic carbons $\left(\mathrm{C}_{\mathrm{AR}}\right)$. The spin density difference for $(\mathbf{a}-\mathbf{c})$ is shown beneath the PDOS $\left(\Delta \rho\left[\mathrm{bohr}^{-1}\right]\right)$, respectively.

\section{Materials and Methods}

The structures herein were investigated using the projector augmented wave (PAW) method within the Vienna Ab Initio Simulation Package (VASP 5.4.4) [63-66]. All calculations are spin-unrestricted and performed using the Perdew-Burke-Ernzerhof (PBE) functional combined with the DFT-D3 van der Waals correction [67-69]. To provide sufficient space for $5 \mathrm{CB}$ on $\mathrm{MV}-\mathrm{G}$, an $8 \times 6$ orthorhombic supercell with a $34 \AA$ out-of-plane vacuum space is used. These comparisons converge as cell sizes increase; thus, we expect small changes in physical properties between the presented orthorhombic cell and the more commonly used hexagonal cell.

Each system is optimized with a cutoff energy of $600 \mathrm{eV}$ and a force tolerance of $0.01 \mathrm{eV} / \AA$. Geometric optimizations are performed at $\Gamma$. These optimizations are followed by band structure, PDOS and binding energy calculations using a $15 \times 15 \times 1 \Gamma$-centered $k$-grid. The binding energy $\left(E_{B}\right)$ is herein defined as

$$
E_{B}=E_{5 C B / M V G}-\left(E_{5 C B}+E_{M V G}\right),
$$

where $E_{5 C B / M V G}, E_{5 C B}$ and $E_{M V G}$ are ground-state energies of the supercell, $5 \mathrm{CB}$ and MV-G, respectively [37]. For the homeotropic scans, 5CB degrees of freedom are free to relax, while MV-G degrees of freedom are frozen. In the planar alignment, 5CB degrees of freedom are frozen to avoid deflection of the molecule away from MV-G and the boundary carbon atoms of MV-G are frozen to prevent nanosheet repulsion, i.e., the nanosheet being pushed down along the negative $z$-axis as $5 \mathrm{CB}$ is brought inside equilibrium distance. For the NEB calculations, we use 19 total images and include the spin-polarized charge density difference for important transitions along the reaction path. See Supplementary Material for the entire set of NEB images and PDOS. The spin-polarized charge density difference is determined from

$$
\Delta \rho=\rho_{5 C B / M V G}-\left(\rho_{5 C B}+\rho_{M V G}\right),
$$

where $\rho_{5 C B} / M V G, \rho_{5 C B}$ and $\rho_{M V G}$ are the total spin-polarized charge densities for the composite system, 5CB and MV-G, respectively.

\section{Conclusions}

Using DFT, we investigated configurations that could lead to $5 \mathrm{CB}$ implantation into a monovacancy defect in MLG. Given the importance of orientation for initiating a reaction, we found homeotropic alignment of $5 \mathrm{CB}$ enabled spontaneous insertion into the mono- 
vacancy. The reaction takes several salient steps: (1) an initial bond with a monovacancy carbon pulls the $5 \mathrm{CB}$ toward the sheet; (2) the cyano-nitrogen forms a tetrahedral bond with the three carbanions in the monovacancy; (3) the resulting under-coordinated cyano-carbon is pulled further into the monovacancy; (4) one of the three monovacancy carbons unbinds the cyano-nitrogen and binds with the cyano-carbon. From the DOS, we can see that the process is mediated by the conjugated biphenyl core of the molecule. The resulting structure stabilizes the monovacancy by inserting both the cyano-carbon and cyano-nitrogen into the sheet, with the biphenyl core aligned normal to the surface. This allows charge to flow between the sheet and the 5CB, shifting the Fermi energy of the graphene.

It is important to note that the homeotropic alignment of the $5 \mathrm{CB}$ molecule on MV-G is dissimilar to the preferred planar orientation on pristine graphene [37]. In the LC phase, this would cause large geometric frustrations in $5 \mathrm{CB}$, which could lead to a weakening of the anchoring energy, a degenerate pretilt at the surface or defects in the LC orientation. On a micro-scale, defect engineering of LC systems has already revealed novel self-assembly, memory effects and control of flow and colloidal motion [70]. Intelligent design of defects such as monovacancies in 2D surfaces would yield a new testbed for examining LC behavior.

Furthermore, our results indicate that defects can play a key role in the interfacial chemistry of LC-based devices incorporating 2D nanosheets. Future work at the intersection of surface vacancies and LC could have broadened significance with respect to patterned defects on 2D nanosheets for application-driven covalent surface functionalization, which could raise new questions concerning tailored interfacial interaction for specific applications.

Supplementary Materials: The following supporting information can be downloaded online, Figure S1: NEB structures for 5CB/MV-G insertion reaction, Figure S2: NEB PDOS for 5CB/MV-G insertion reaction.

Author Contributions: Conceptualization, P.A.B. and D.G.; Funding acquisition, C.M.S.; Investigation, P.A.B.; Project administration, D.G.; Supervision, S.A.F. and D.G.; Visualization, P.A.B. and J.K.; Writing—original draft, P.A.B.; Writing—review \& editing, J.K., S.A.F., C.M.S. and D.G. All authors have read and agreed to the published version of the manuscript.

Funding: This research received no external funding.

Institutional Review Board Statement: Not applicable.

Informed Consent Statement: Not applicable.

Data Availability Statement: The data presented in this study are available on request from the corresponding author. The data are not publicly available due to U.S. Department of Defense technical information controls.

Acknowledgments: This work has been supported by the Office of Naval Research through the U.S. Naval Research Laboratory. P.A.B. acknowledges the National Research Council Research Associateship Programs.

Conflicts of Interest: The authors declare no conflict of interest.

Sample Availability: Not available.

\section{References}

1. Yu, J.S.; Lev, B.; Jin, H.; Park, K.; Chae, D.H.; Kim, W.S.; Kim, J.H. Surface-induced transition of nematic liquid crystals on graphene/SiC substrate. EPL 2018, 124, 46004. [CrossRef]

2. Yu, J.S.; Yun, J.E.; Kim, J.-H.H. Tilt angle change of nematic liquid crystal as a function of the number of graphite layers. Liq. Cryst. 2013, 40, 216-220. [CrossRef]

3. Shen, T.Z.; Hong, S.H.; Lee, J.H.; Kang, S.G.; Lee, B.; Whang, D.; Song, J.K. Selectivity of Threefold Symmetry in Epitaxial Alignment of Liquid Crystal Molecules on Macroscale Single-Crystal Graphene. Adv. Mater. 2018, 30, 1802441. [CrossRef] [PubMed] 
4. Basu, R.; Lee, A. Ion trapping by the graphene electrode in a graphene-ITO hybrid liquid crystal cell. Appl. Phys. Lett. 2017, 111, 5-9. [CrossRef]

5. Lim, Y.J.; Lee, B.H.; Kwon, Y.R.; Choi, Y.E.; Murali, G.; Lee, J.H.; Nguyen, V.L.; Lee, Y.H.; Lee, S.H. Monitoring defects on monolayer graphene using nematic liquid crystals. Opt. Express 2015, 23, 14162. [CrossRef]

6. Son, J.-H.; Baeck, S.-J.; Park, M.-H.; Lee, J.-B.; Yang, C.-W.; Song, J.-K.; Zin, W.-C.; Ahn, J.-H. Detection of graphene domains and defects using liquid crystals. Nat. Commun. 2014, 5, 3484. [CrossRef]

7. Woo Kim, D.; Ho Kim, Y.; Su Jeong, H.; Jung, H.-T. Direct visualization of large-area graphene domains and boundaries by optical birefringency. Nat. Nanotechnol. 2012, 7, 29-34. [CrossRef]

8. Naqvi, B.A.; Shehzad, M.A.; Cha, J.; Min, K.-A.; Khan, M.F.; Hussain, S.; Seo, Y.; Hong, S.; Eom, J.; Jung, J. Visualizing Degradation of Black Phosphorus Using Liquid Crystals. Sci. Rep. 2018, 8, 12966. [CrossRef]

9. Basu, R.; Kinnamon, D.; Spillmann, C.M.; Kolacz, J.; Frantz, J.A.; Myers, J.D. Graphene as an Alignment Layer and Electrode for Liquid Crystal Devices. U.S. Patent 10,564,505, 18 February 2020.

10. Basu, R.; Shalov, S.A. Graphene as transmissive electrodes and aligning layers for liquid-crystal-based electro-optic devices. Phys. Rev. E 2017, 96, 012702. [CrossRef]

11. Basu, R.; Garvey, A.; Kinnamon, D. Effects of graphene on electro-optic response and ion-transport in a nematic liquid crystal. J. Appl. Phys. 2015, 117, 074301. [CrossRef]

12. Rummeli, M.H.; Ta, H.Q.; Mendes, R.G.; Gonzalez-Martinez, I.G.; Zhao, L.; Gao, J.; Fu, L.; Gemming, T.; Bachmatiuk, A.; Liu, Z. New Frontiers in Electron Beam-Driven Chemistry in and around Graphene. Adv. Mater. 2019, 31, 1800715. [CrossRef] [PubMed]

13. Jiang, J.; Xu, T.; Lu, J.; Sun, L.; Ni, Z. Defect Engineering in 2D Materials: Precise Manipulation and Improved Functionalities. Research 2019, 2019, 1-14. [CrossRef] [PubMed]

14. Lin, Z.; Carvalho, B.R.; Kahn, E.; Lv, R.; Rao, R.; Terrones, H.; Pimenta, M.A.; Terrones, M. Defect engineering of two-dimensional transition metal dichalcogenides. 2D Mater. 2016, 3, 022002. [CrossRef]

15. Geim, A.K.; Grigorieva, I.V. Van der Waals heterostructures. Nature 2013, 499, 419-425. [CrossRef]

16. Miró, P.; Audiffred, M.; Heine, T. An atlas of two-dimensional materials. Chem. Soc. Rev. 2014, 43, 6537-6554. [CrossRef]

17. Bhimanapati, G.R.; Lin, Z.; Meunier, V.; Jung, Y.; Cha, J.; Das, S.; Xiao, D.; Son, Y.; Strano, M.S.; Cooper, V.R.; et al. Recent Advances in Two-Dimensional Materials beyond Graphene. ACS Nano 2015, 9, 11509-11539. [CrossRef]

18. Novoselov, K.S.; Mishchenko, A.; Carvalho, A.; Castro Neto, A.H. 2D materials and van der Waals heterostructures. Science 2016, 353, aac9439. [CrossRef]

19. Fiori, G.; Bonaccorso, F.; Iannaccone, G.; Palacios, T.; Neumaier, D.; Seabaugh, A.; Banerjee, S.K.; Colombo, L. Electronics based on two-dimensional materials. Nat. Nanotechnol. 2014, 9, 768-779. [CrossRef]

20. Schwierz, F.; Pezoldt, J.; Granzner, R. Two-dimensional materials and their prospects in transistor electronics. Nanoscale 2015, 7 , 8261-8283. [CrossRef]

21. Deng, D.; Novoselov, K.S.; Fu, Q.; Zheng, N.; Tian, Z.; Bao, X. Catalysis with two-dimensional materials and their heterostructures. Nat. Nanotechnol. 2016, 11, 218-230. [CrossRef]

22. Fu, Q.; Bao, X. Surface chemistry and catalysis confined under two-dimensional materials. Chem. Soc. Rev. 2017, 46, 1842-1874. [CrossRef] [PubMed]

23. Chia, X.; Pumera, M. Characteristics and performance of two-dimensional materials for electrocatalysis. Nat. Catal. 2018, 1, 909-921. [CrossRef]

24. Mendoza-Sánchez, B.; Gogotsi, Y. Synthesis of Two-Dimensional Materials for Capacitive Energy Storage. Adv. Mater. 2016, 28, 6104-6135. [CrossRef]

25. Peng, L.; Zhu, Y.; Chen, D.; Ruoff, R.S.; Yu, G. Two-Dimensional Materials for Beyond-Lithium-Ion Batteries. Adv. Energy Mater. 2016, 6, 1600025. [CrossRef]

26. Ren, Y.; Qiao, Z.; Niu, Q. Topological phases in two-dimensional materials: A review. Rep. Prog. Phys. 2016, 79, 066501. [CrossRef] [PubMed]

27. Wang, A.Q.; Ye, X.G.; Yu, D.P.; Liao, Z.M. Topological Semimetal Nanostructures: From Properties to Topotronics. ACS Nano 2020, 14, 3755-3778. [CrossRef] [PubMed]

28. Sun, Z.; Chang, H. Graphene and graphene-like two-dimensional materials in photodetection: Mechanisms and methodology. ACS Nano 2014, 8, 4133-4156. [CrossRef]

29. Sun, Z.; Martinez, A.; Wang, F. Optical modulators with 2D layered materials. Nat. Photonics 2016, 10, 227-238. [CrossRef]

30. Xia, F.; Wang, H.; Xiao, D.; Dubey, M.; Ramasubramaniam, A. Two-dimensional material nanophotonics. Nat. Photonics 2014, 8 , 899-907. [CrossRef]

31. Zhao, H.; Guo, Q.; Xia, F.; Wang, H. Two-dimensional materials for nanophotonics application. Nanophotonics 2015, 4, 128-142. [CrossRef]

32. Mak, K.F.; Shan, J. Photonics and optoelectronics of 2D semiconductor transition metal dichalcogenides. Nat. Photonics 2016, 10, 216-226. [CrossRef]

33. Schwartz, J.J.; Mendoza, A.M.; Wattanatorn, N.; Zhao, Y.; Nguyen, V.T.; Spokoyny, A.M.; Mirkin, C.A.; Baše, T.; Weiss, P.S. Surface dipole control of liquid crystal alignment. J. Am. Chem. Soc. 2016, 138, 5957-5967. [CrossRef] [PubMed] 
34. Dyck, O.; Zhang, L.; Yoon, M.; Swett, J.L.; Hensley, D.; Zhang, C.; Rack, P.D.; Fowlkes, J.D.; Lupini, A.R.; Jesse, S. Doping transition-metal atoms in graphene for atomic-scale tailoring of electronic, magnetic, and quantum topological properties. Carbon N. Y. 2021, 173, 205-214. [CrossRef]

35. Shehzad, M.A.; Tien, D.H.; Iqbal, M.W.; Eom, J.; Park, J.H.; Hwang, C.; Seo, Y. Nematic Liquid Crystal on a Two Dimensional Hexagonal Lattice and its Application. Sci. Rep. 2015, 5, 13331. [CrossRef]

36. Shehzad, M.A.; Lee, J.; Park, S.H.; Akhtar, I.; Khan, M.F.; Hussain, S.; Eom, J.; Jung, J.; Kim, G.; Hwang, C.; et al. Dynamics of liquid crystal on hexagonal lattice. 2D Mater. 2018, 5, 045021. [CrossRef]

37. Fischer, S.A.; Kołacz, J.; Spillmann, C.M.; Gunlycke, D. Adsorption of the liquid crystal molecule 5CB on graphene. Phys. Rev. E 2018, 98, 052702. [CrossRef]

38. Brown, P.A.; Fischer, S.A.; Kołacz, J.; Spillmann, C.; Gunlycke, D. Thermotropic liquid crystal (5CB) on two-dimensional materials. Phys. Rev. E 2019, 100, 62701. [CrossRef]

39. Stone, A.J.; Wales, D.J. Theoretical studies of icosahedral C60 and some related species. Chem. Phys. Lett. 1986, 128, 501-503. [CrossRef]

40. Thrower, P.A. Chemistry E Physics of Carbon: Volume 25; CRC Press: Boca Raton, FL, USA, 1996.

41. $\mathrm{Wu}, \mathrm{L} . ; \mathrm{Hu}, \mathrm{S}$.; Yu, W.; Shen, S.; Li, T. Stabilizing mechanism of single-atom catalysts on a defective carbon surface. NPJ Comput. Mater. 2020, 6, 23. [CrossRef]

42. Carr, L.D.; Lusk, M.T. Defect engineering: Graphene gets designer defects. Nat. Nanotechnol. 2010, 5, 316-317. [CrossRef]

43. Kotakoski, J.; Krasheninnikov, A.V.; Kaiser, U.; Meyer, J.C. From point defects in graphene to two-dimensional amorphous carbon. Phys. Rev. Lett. 2011, 106, 105505. [CrossRef] [PubMed]

44. Banhart, F.; Kotakoski, J.; Krasheninnikov, A.V. Structural defects in graphene. ACS Nano 2011, 5, 26-41. [CrossRef] [PubMed]

45. Yazyev, O.V.; Chen, Y.P. Polycrystalline graphene and other two-dimensional materials. Nat. Nanotechnol. 2014, 9, 755-767. [CrossRef] [PubMed]

46. Rasool, H.I.; Ophus, C.; Zettl, A. Atomic Defects in Two Dimensional Materials. Adv. Mater. 2015, 27, 5771-5777. [CrossRef] [PubMed]

47. Zhang, H.; Lv, R. Defect engineering of two-dimensional materials for efficient electrocatalysis. J. Mater. 2018, 4, 95-107. [CrossRef]

48. Brown, P.A.; Xu, C.; Shuford, K.L. Periodic trends of pnictogen substitution into a graphene monovacancy: A first-principles investigation. Chem. Mater. 2014, 26, 5735-5744. [CrossRef]

49. Liu, Y.; Xiao, C.; Li, Z.; Xie, Y. Vacancy Engineering for Tuning Electron and Phonon Structures of Two-Dimensional Materials. Adv. Energy Mater. 2016, 6, 1600436. [CrossRef]

50. Xu, C.; Brown, P.A.; Lu, J.; Shuford, K.L. Electronic Properties of Halogen-Adsorbed Graphene. J. Phys. Chem. C 2015, 119, 17271-17277. [CrossRef]

51. Jia, Y.; Zhang, L.; Du, A.; Gao, G.; Chen, J.; Yan, X.; Brown, C.L.; Yao, X. Defect Graphene as a Trifunctional Catalyst for Electrochemical Reactions. Adv. Mater. 2016, 28, 9532-9538. [CrossRef]

52. Grasseschi, D.; Silva, W.C.; de Souza Paiva, R.; Starke, L.D.; do Nascimento, A.S. Surface coordination chemistry of graphene: Understanding the coordination of single transition metal atoms. Coord. Chem. Rev. 2020, 422, 213469. [CrossRef]

53. Ding, Y.; Luo, S.; Ma, L.; An, J. Reductive Cleavage of Unactivated Carbon-Cyano Bonds under Ammonia-Free Birch Conditions J. Org. Chem. 2019, 84, 15827-15833. [CrossRef] [PubMed]

54. Thevenin, L.; Fliedel, C.; Fantin, M.; Ribelli, T.G.; Matyjaszewski, K.; Poli, R. Reductive Termination of Cyanoisopropyl Radicals by Copper(I) Complexes and Proton Donors: Organometallic Intermediates or Coupled Proton-Electron Transfer? Inorg. Chem. 2019, 58, 6445-6457. [CrossRef] [PubMed]

55. Tsai, Y.J.; Kuo, C.L. Effect of Structural Disorders on the Li Storage Capacity of Graphene Nanomaterials: A First-Principles Study. ACS Appl. Mater. Interfaces 2020, 12, 22917-22929. [CrossRef] [PubMed]

56. Robertson, A.W.; Do Lee, G.; He, K.; Yoon, E.; Kirkland, A.I.; Warner, J.H. The role of the bridging atom in stabilizing odd numbered graphene vacancies. Nano Lett. 2014, 14, 3972-3980. [CrossRef]

57. Rodrigo, L.; Pou, P.; Pérez, R. Graphene monovacancies: Electronic and mechanical properties from large scale ab initio simulations. Carbon N. Y. 2016, 103, 200-208. [CrossRef]

58. Jiang, Q.G.; Ao, Z.M.; Zheng, W.T.; Li, S.; Jiang, Q. Enhanced hydrogen sensing properties of graphene by introducing a mono-atom-vacancy. Phys. Chem. Chem. Phys. 2013, 15, 21016-21022. [CrossRef]

59. Chigo Anota, E.; Escobedo-Morales, A.; Salazar Villanueva, M.; Vázquez-Cuchillo, O.; Rubio Rosas, E. On the influence of point defects on the structural and electronic properties of graphene-like sheets: A molecular simulation study. J. Mol. Model. 2013, 19, 839-846. [CrossRef]

60. Zhang, Y.; Gao, F.; Gao, S.; He, L. Tunable magnetism of a single-carbon vacancy in graphene. Sci. Bull. 2020, 65, 194-200 [CrossRef]

61. Wang, X.C.; Zhang, Y.; Liu, F.C.; Ma, Y.; Feng, W.; Zhang, S.X.A. Dynamic nano-pulling effect of the boron-functionalized graphene monovacancy for molecule dissociation. J. Phys. D Appl. Phys. 2013, 46, 385302. [CrossRef]

62. Yu, L.; Du, C.; Liu, X. Dy adsorption and penetration on defected graphene by first-principles calculations. Mater. Res. Express 2018, 5, 025022. [CrossRef]

63. Blöchl, P.E. Projector augmented-wave method. Phys. Rev. B 1994, 50, 17953-17979. [CrossRef] [PubMed] 
64. Hafner, J. Materials simulations using VASP-a quantum perspective to materials science. Comput. Phys. Commun. 2007, 177, 6-13. [CrossRef]

65. Hafner, J. Ab-Initio Simulations of Materials Using VASP: Density-Functional Theory and Beyond. J. Comput. Chem. 2008, 177, 2044-2078. [CrossRef] [PubMed]

66. Joubert, D. From ultrasoft pseudopotentials to the projector augmented-wave method. Phys. Rev. B Condens. Matter Mater. Phys. 1999, 59, 1758-1775. [CrossRef]

67. Perdew, J.P.; Burke, K.; Ernzerhof, M. Generalized gradient approximation made simple. Phys. Rev. Lett. 1996, 77, 3865-3868. [CrossRef] [PubMed]

68. Paier, J.; Hirschl, R.; Marsman, M.; Kresse, G. The Perdew-Burke-Ernzerhof exchange-correlation functional applied to the G2-1 test set using a plane-wave basis set. J. Chem. Phys. 2005, 122, 234102. [CrossRef] [PubMed]

69. Grimme, S.; Antony, J.; Ehrlich, S.; Krieg, H. A consistent and accurate ab initio parametrization of density functional dispersion correction (DFT-D) for the 94 elements H-Pu. J. Chem. Phys. 2010, 132, 154104. [CrossRef] [PubMed]

70. Araki, T.; Serra, F.; Tanaka, H. Defect science and engineering of liquid crystals under geometrical frustration. Soft Matter 2013, 9, 8107-8120. [CrossRef] 